\title{
1-(Aminomethyl)-trans-cyclooct-4-ene, a new TCO derivative for target visualization and target engagement measurement in cells
} \author{
Schultz ${ }^{a, d^{*}}$ and Giovanna Bergamini ${ }^{b^{*}}$ \\ a European Molecular Biology Laboratory (EMBL), Heidelberg, Germany. \\ b Cellzome, a GlaxoSmithKline company, Heidelberg, Germany. \\ c GlaxoSmithKline (GSK) Medicines Research Centre, Stevenage, United Kingdom. \\ d Oregon Health \& Science University (OHSU), Portland, OR, USA. \\ *Corresponding authors: schultz@embl.de, giovanna.2.bergamini@gsk.com
}

Cécile Echalier, ${ }^{\text {a,b }}$ Anna Rutkowska, ${ }^{b}$ Douglas W. Thomson, ${ }^{b}$ Lee J. Edwards, ${ }^{c}$ Blandine S. J. McKay, ${ }^{c}$ Marcel Mülbaier, ${ }^{b}$ Carsten

\begin{abstract}
A new trans-cyclooctene derivative, amTCO, was synthesised using a phthalimide protecting group as a built-in photosensitiser for the cyclooctene isomerization. An amTCO probe targeting indoleamine 2,3-dioxygenase (IDO1) was superior to a TCO variant. The new probe is an effective tool for visualizing IDO1 and measuring the binding affinities of small molecule inhibitors to IDO1 in cells.
\end{abstract}

The increasing costs and risks associated with the development of a new therapeutic drug are a major challenge for the pharmaceutical industry. ${ }^{1}$ Insufficient target engagement and adverse effects caused by off-target activities contribute to drug failure in late stages of development. ${ }^{2}$ To enhance the success of drug discovery, a comprehensive characterization of drug interactions at the preclinical stage is essential. Recent developments in click chemistry have greatly facilitated such studies, enabling drug visualization and measurement of drugtarget binding in complex biological systems. ${ }^{3}$

Click chemistry refers to reactions with a high thermodynamic force that proceed quickly to completion in an aqueous environment, generating a single reaction product in high yield. ${ }^{4}$ Click probes derived from drugs are obtained by functionalization with a clickable moiety, enabling conjugation via a selective reaction between the click probe and a chosen reporter in a relevant disease model. In previous work, we demonstrated that this strategy can be used to identify drug targets and off-targets, visualise the cellular localization of the probe and measure target engagement of non-derivatised drug compounds. ${ }^{5}$ In the same study, different click reactions were compared and the inverse electron demand Diels Alder (IEDDA) reaction between a trans-cyclooctene (TCO) and a tetrazine was identified as the most efficient. The superior performance of the IEDDA reaction in our click probe strategy can be attributed to its unmatchable kinetics. $^{6}$ Indeed, endogenous concentrations of target proteins can be too low for robust detection. Consequently, ultrafast kinetics not only reduce unspecific reactions but ensure a complete reaction between the click probe and the reporter under diluted conditions on a reasonable time scale. The selectivity and high reactivity of TCO with tetrazine, combined with its prolonged stability in aqueous solutions, makes it a very attractive click moiety. Nevertheless, TCO is a hydrophobic group. When attached to a drug, it may affect the physical chemical properties of the click probe as well as the pharmacological properties for the drug. In this context, considerable efforts have been made by several research groups to develop new trans-cyclooctene derivatives with reduced hydrophobicity (e.g. oxoTCO) ${ }^{7}$ or faster kinetics (e.g. sTCO, dTCO). ${ }^{8,9}$

Herein, we report a new trans-cyclooctene derivative, the 1(aminomethyl)-trans-cyclooct-4-ene (amTCO), which differs from the original trans-cyclooct-4-enol by the functional group used for conjugation. This new derivative was applied to the preparation of a click probe targeting the indoleamine 2,3dioxygenase (IDO1). We demonstrate here that amTCO results in a reduction of hydrophobicity as compared to the previously described TCO-based GSK5112 click probe, ${ }^{10}$ and in a reduction of background in imaging-based readouts. This allows the new probe to be used for measuring binding affinities of unmodified IDO1 inhibitors in cells.

TCO probes are traditionally obtained by coupling transcyclooct-4-enol (TCO-OH) onto an amine-containing compound. $\mathrm{TCO}-\mathrm{OH}$ is commercially available as a pre-activated paranitrophenol or $\mathrm{N}$-hydroxysuccinimide ester which makes the coupling very straightforward. However, for carboxylic acid or hydroxyl-containing compounds, direct coupling of $\mathrm{TCO}-\mathrm{OH}$ would result in an ester or carbonate linkage, both hydrolysable. Therefore, in this case, TCO with a linkable amine would be more convenient for stable conjugation. Furthermore, we hypothesised that a different linkage between the TCO and the drug could help tuning the physical chemical properties of the probes. We calculated octanol/water partition coefficients ( $\log P$ values) for a click probe obtained by coupling amTCO onto a carboxylic acid (amide linkage) and found the amTCO derivative less hydrophobic than a click probe obtained by coupling $\mathrm{TCO}-\mathrm{OH}$ onto an amine (carbamate linkage) (Figure 1).

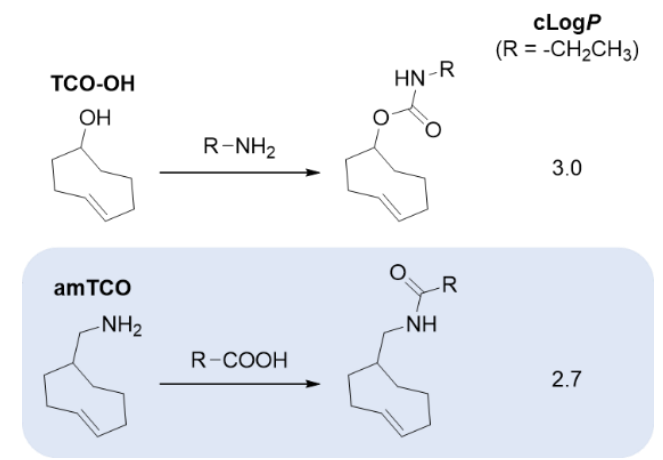

Figure 1. Structures of TCO-OH and amTCO, and calculated log P values (cLog P) for TCO-OH and amTCO conjugates. 
A

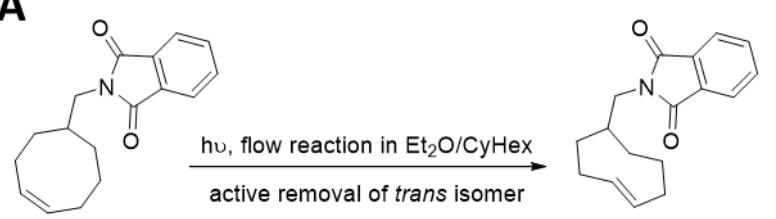

B

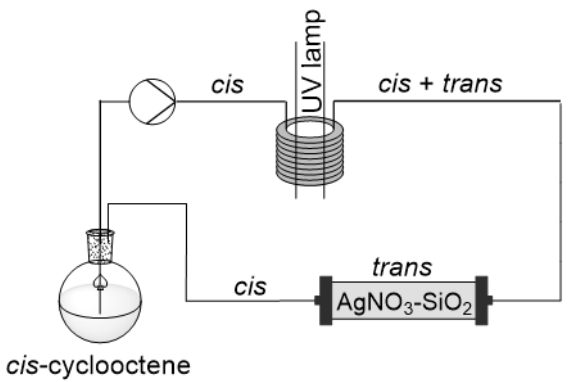

C

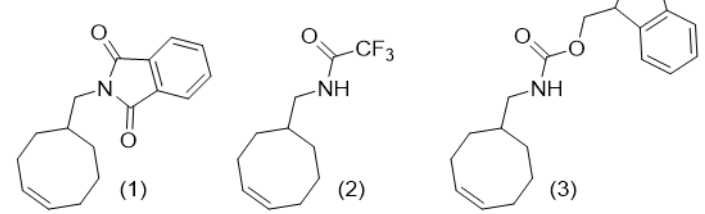

\begin{tabular}{cccc}
\hline Compound & $\begin{array}{c}\text { Additional } \\
\text { photosensitizer (1 eq.) }\end{array}$ & Irradiation time & $\begin{array}{c}\text { Conversion } \\
\text { cis } \rightarrow \text { trans }\end{array}$ \\
\hline (1) & - & $65 \mathrm{~min}$ & $75 \%$ \\
(2) & Methyl benzoate & $85 \mathrm{~min}$ & $70 \%$ \\
(2) & Dimethyl isophthalate & $65 \mathrm{~min}$ & $80 \%$ \\
(3) & Dimethyl isophthalate & $90 \mathrm{~min}$ & $40 \%$ \\
\hline
\end{tabular}

Figure 2. (A) Photoisomerization of amCCO into amTCO using a phthalimide protecting group as intramolecular photosensitiser. (B) Photoi somerization setup for irradiation of ciscyclooctenes at $254 \mathrm{~nm}$ in continuous flow with trapping of trans-cyclooctenes on silver nitrate-coated silica. (C) Comparative study of different photoisomerization conditions.

amTCO was prepared from commercially available 1(aminomethyl)-cis-cyclooct-4-ene (amcCO) (Figure 2, A). The photoisomerisation was conducted in a closed-loop flow reactor similar to the setup developed by Svatunek et al. (Figure 2 , B). ${ }^{11}$ The cis isomer was irradiated in continuous flow in the presence of a photosensitiser. It is expected that after each cycle of irradiation less than half of the cis cyclooctene is converted into the corresponding trans. In order to drive the photoisomerization to completion, the trans isomer was trapped on $\mathrm{AgNO}_{3}$-impregnated silica while the remaining cis isomer was irradiated further. ${ }^{12}$ It is important to ensure that the cis derivative is eluted from the silica column and returns into the reservoir whereas the trans-cyclooctene- $\mathrm{AgNO}_{3}$ complex remains adsorbed onto silica. Therefore, a protecting group had to be introduced onto amCCO in order to decrease its polarity. In the first instance, the amine was protected as a trifluoroacetamide and methyl benzoate was used as a photosensitiser following a standard approach. ${ }^{11,12}$ The reaction was monitored by measuring the remaining cis derivative by gas chromatography. This procedure resulted in $70 \%$ cis-to-trans conversion after $85 \mathrm{~min}$ irradiation (Figure 2, C). Although methyl benzoate is the most commonly used photosensitiser for cyclooctene isomerization, ${ }^{11,12}$ dimethyl isophtalate has the potential to give higher trans/cis ratios in the photostationary state. ${ }^{13}$ As expected, a higher conversion in a shorter irradiation time was observed when methyl benzoate was replaced by dimethyl isophthalate. Based on a similarity in structure, we hypothesised that a phtalimide used as a protecting group could act as a built-in sensitiser. Accordingly, phthalimide-protected amCCO (1) was successfully converted into phthalimideprotected amTCO when irradiated in the absence of any additional photosensitiser. The isomerization proceeded at a similar rate as trifluoroacetamide-amTCO (2) with dimethyl isophthalate. Hence, it was more efficient than the standard procedure using methyl benzoate as a photosensitiser. In addition, the reaction could conveniently be monitored by HPLC as the phthalimide group is UV-active. As a control, Fmoc- protected amCCO (3) was also irradiated in the set up. As expected, strong absorption was observed but no trans product was detected. This suggests that the Fmoc protecting group absorbed light but did not transfer energy to the double bond. In the presence of dimethyl isophthalate, the photoisomerisation of Fmoc-amCCO (3) took place with low efficiency likely because most of the light was absorbed by the Fmoc group rather than the photosensitiser. Therefore, phthalimides are useful protecting groups for the photoisomerization of primary amine-containing cyclooctenes. They can photosensitise the reaction efficiently and independently of the concentration in the reaction mixture.

As proof-of-concept, amTCO was used for the preparation of a click probe targeting the indoleamine 2,3-dioxygenase (IDO1). Reports have shown that increased IDO1 activity promotes an immunosuppressive microenvironment that is believed to support tumour growth. ${ }^{14}$ Therefore, it has been considered for cancer immunotherapies. IDO1 is an intracellular enzyme involved in tryptophan catabolism and requires heme binding for its activity. Due to this property, there is a high need for tools that enable the preclinical evaluation of IDO1 inhibitors in living cells under endogenous conditions. In this context, amTCO was coupled to a linkable analog of the IDO1 inhibitor GSK5628 (Figure 3). The new probe, amTCO-GSK9353, was compared to a previously reported TCO probe targeting IDO1, TCOGSK5112. ${ }^{10}$ The log D (distribution coefficient at $\mathrm{pH}$ 7.4) of the two probes was measured by chromatography and used as an indicator of their lipophilicity. The new amTCO group in combination with the short additional linker resulted in a 2-log reduction of the log $D$ value (Figure 3 ). This decrease in lipophilicity was expected to have a positive impact on the performance of the probe.

Both probes, amTCO-GSK9353 and TCO-GSK5112, were first compared in an imaging assay to visualise their subcellular localization. IDO1 expression was induced in HeLa cells using human interferon-gamma (IFNY). Live cells were treated with amTCO-GSK9353 and TCO-GSK5112 probes at $3 \mu \mathrm{M}$ final 

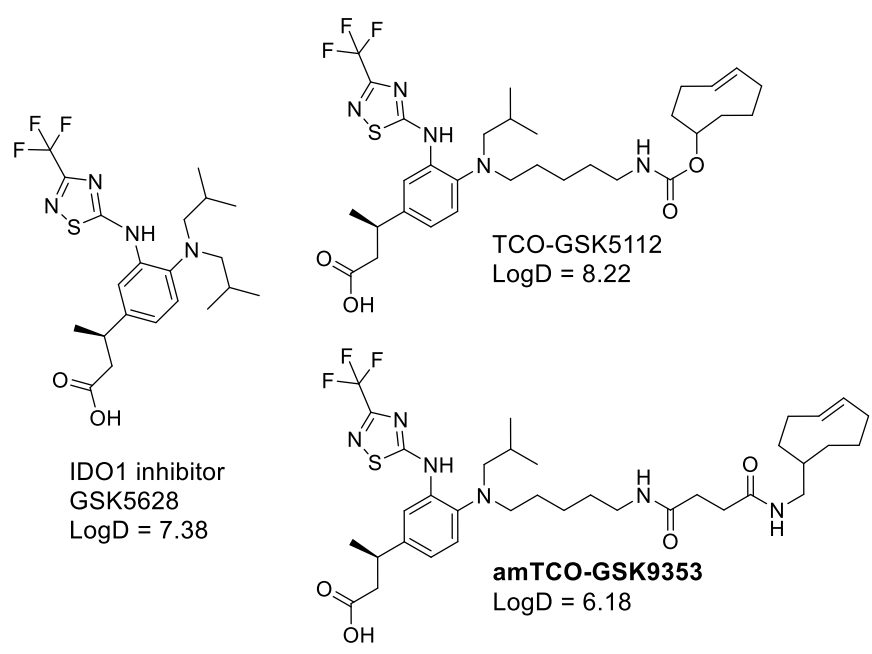

Figure 3. Structure and log D value of the parent compound GSK5628 and derived click probes.

concentration for 1 hour. After fixation and permeabilization of the cells, a dye reporter (Cy5-tetrazine) was added in order to visualise the localization of the probes by confocal fluorescence microscopy. As expected, both probes accumulated in the nucleus and the cytoplasm in agreement with the reported localization of IDO1 (Figure 4, A). However, in addition to this signal, we observed accumulation of TCO-GSK5112 probe in the endoplasmic reticulum (ER) and Golgi, especially in cells where the levels of IDO1 protein were low (Figure 4, A, white arrows). This accumulation, most probably driven by the high lipophilicity of the probe, was also visible in a competitive binding assay where each probe $(3 \mu \mathrm{M})$ and the parent compound GSK5628 (10 $\mu \mathrm{M})$ were simultaneously added to the induced cells and therefore competed for the same binding site in IDO1. Due to the excess of competitor, specific binding of both probes was suppressed. However, significant residual fluorescent signal in ER/Golgi could still be detected for TCOGSK5112, confirming superior quality of the newly designed amTCO-GSK9353.

In order to quantify the unspecific accumulation of the probes, IFNY-stimulated HeLa cells were treated with the probes and the parent inhibitor GSK5628, trypsinised and analysed by FACS after staining (Figure 4, B). In the absence of competitor, both probes gave a fluorescent signal of similar intensity. However, only partial competition (80\%) was observed for TCO-GSK5112 while total competition (100\%) was achieved with amTCOGSK9353. These results confirmed the confocal microscopy observations. The unspecific accumulation of TCO-GSK5112 was so far preventing its use at high concentration. With its reduced
A
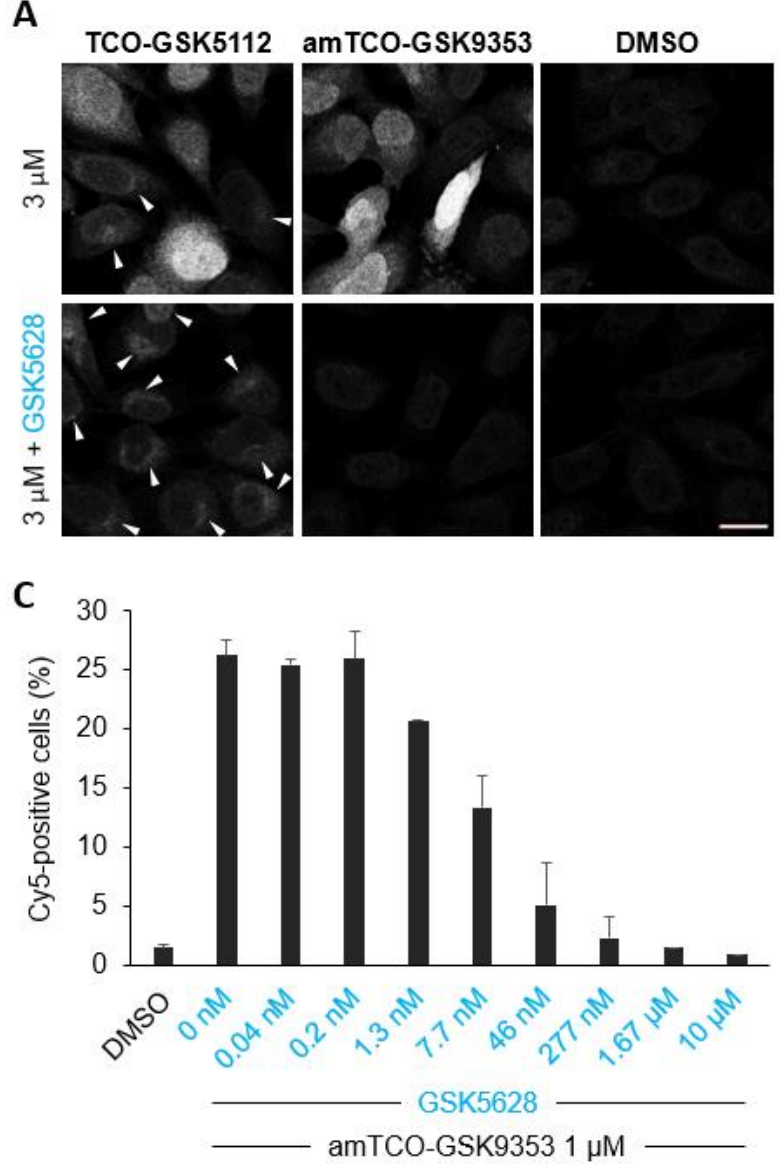

B

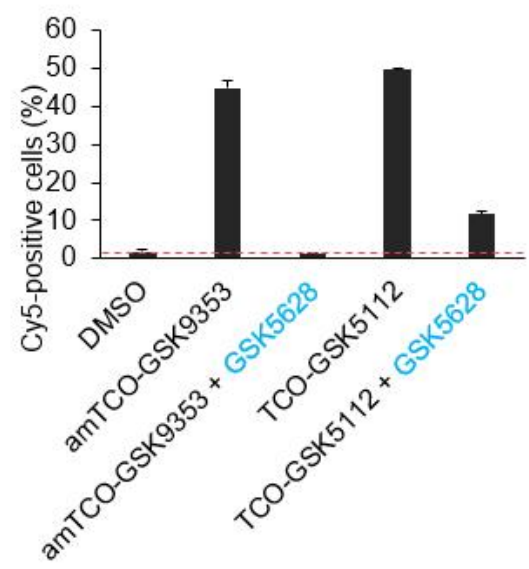

D

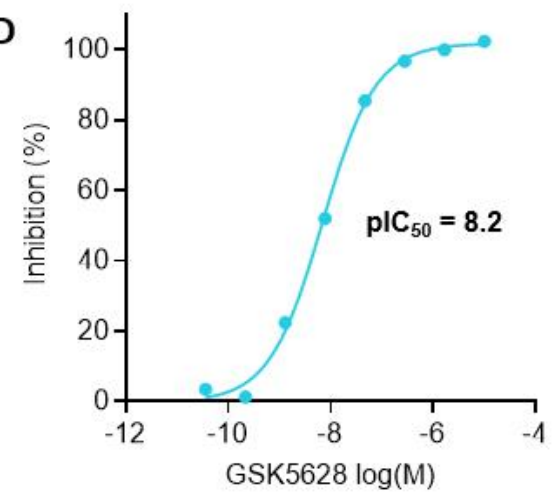

Figure 4. (A) Imaging assay. IFN $y$-treated HeLa cells were incubated with $3 \mu \mathrm{M}$ click probe \pm GSK5628 for $1 \mathrm{~h}$, followed by fixation, permeabilization and click reaction with 200 $\mathrm{nM}$ Cy5-Tetrazine. Representative fluorescent images recorded after excitation at $633 \mathrm{~nm}(\mathrm{Cy} 5)$ are shown. Scale bar $=20 \mu \mathrm{m}$. The white arrows point at the unspecific accumulation of TCO-GSK5112. (B) Quantification of Cy5 fluorescence signal in HeLa cells treated as in A and analysed by flow cytometry. Dashed line shows DMSO level. Data from one representative experiment (replicates shown in Figure S14). (C) Flow cytometric analysis of IFN $\gamma$-induced HeLa cells treated with $1 \mu \mathrm{M}$ amTCO-GSK9353 and increasing concentrations of GSK5628, followed by trypsinization, fixation, permeabilization and click reaction with Cy5-Tetrazine. Data from one representative experiment (replicates shown in Figure S16). (D) Competition binding curve of GSK5628 derived from flow cytometric data presented in C. 
hydrophobicity, amTCO-GSK9353 can be employed over a wide concentration range which clearly increases its application spectrum.

One of the most important applications of click probes is measurement of the direct binding of an unlabelled drug to its target in live cells. ${ }^{5}$ Such target engagement measurements aim to confirm that a compound interacts with its intended biological target and to quantify this interaction. This characterization is fundamental in medicinal chemistry to minimise failure of drug candidates at a late stage of development. Briefly, a dose-response relationship is obtained when cells are treated with a fixed concentration of click probe and increasing concentrations of the drug of interest. The $\mathrm{IC}_{50}$ value of the drug is directly inferred from the dose-response curve. This approach was applied to our unlabelled IDO1 inhibitor GSK5628 using amTCO-GSK9353 probe. The residual fluorescent signal was measured by FACS for each concentration of GSK5628 tested in HeLa cells (Figure 4, C). A $\mathrm{pIC}_{50}$ of $8.2\left(\mathrm{IC}_{50}=6.88 \mathrm{nM}\right)$ was determined for the IDO1 inhibitor (Figure 4, D), in line with the affinity measured in an activity-based assay where accumulation of kynurenine was quantified in HeLa cells $\left(\mathrm{pIC}_{50}=8.2\right) \cdot{ }^{10}$

To summarise, we demonstrated that a phthalimide protecting group can conveniently be used as a built-in photosensitiser for the photoisomerisation of trans-cyclooctenes. We introduced a new trans-cyclooctene derivative, amTCO, that features a primary amine for stable conjugation to carboxylic acids and alcohols. We showed that amTCO can help tuning the physical chemical properties of resultant click probes, particularly when the liphophilicity of the parent compound is critical. As an example, a new click probe targeting IDO1 was synthesised with much higher specificity and better performance than the existing tool. We were able to visualise the localisation of IDO1 in cells and measure target engagement of unmodified IDO1 inhibitors. This new tool allows the assessment of IDO1 inhibitors in cellular models. Beyond this work, amTCO could be applied to the preparation of other probes for intracellular click chemistry but also for labelling of biomolecules such as proteins and nucleic acids.

\section{Conflicts of interest}

C.E. worked under an agreement between EMBL, Cellzome GmbH and GlaxoSmithKline. A.R, D.T., L.E, B.M, M.M and G.B. are employees and/or shareholders of Cellzome $\mathrm{GmbH}$ and/or GlaxoSmithKline. C.S. is a consultant and a shareholder of SiChem $\mathrm{GmbH}$. The remaining authors declare no competing financial interests.

\section{Acknowledgements}

We acknowledge the support of the EMBL Metabolomics Core Facility (MCF) in the acquisition and analysis of high resolution mass spectrometry data.

\section{Notes and references}

1 J. A. DiMasi, H. G. Grabowski and R. W. Hansen, J. Health Econ., 2016, 47, 20-33.

2 D. A. Parasrampuria, L. Z. Benet and A. Sharma, AAPS J., 2018, 20, 46 .

3 P. Thirumurugan, D. Matosiuk and K. Jozwiak, Chem. Rev., 2013, 113, 4905-4979.

4 H. C. Kolb, M. G. Finn and K. B. Sharpless, Angew. Chem. Int. Ed., 2001, 40, 2004-2021.

5 A. Rutkowska, D. W. Thomson, J. Vappiani, T. Werner, K. M. Mueller, L. Dittus, J. Krause, M. Muelbaier, G. Bergamini and M. Bantscheff, ACS Chem. Biol., 2016, 11, 2541-2550.

6 B. L. Oliveira, Z. Guo and G. J. L. Bernardes, Chem. Soc. Rev., 2017, 46, 4895-4950.

7 W. D. Lambert, S. L. Scinto, O. Dmitrenko, S. J. Boyd, R. Magboo, R. A. Mehl, J. W. Chin, J. M. Fox and S. Wallace, Org. Biomol. Chem., 2017, 15, 6640-6644.

8 M. T. Taylor, M. L. Blackman, O. Dmitrenko and J. M. Fox, J. Am. Chem. Soc., 2011, 133, 9646-9649.

9 A. Darko, S. Wallace, O. Dmitrenko, M. M. Machovina, R. A. Mehl, J. W. Chin and J. M. Fox, Chem Sci, 2014, 5, 3770-3776.

10 R. Ortiz-Meoz, L. Wang, R. Matico, A. Rutkowska, M. D. la Rosa, S. Bedard, R. Midgett, K. Strohmer, D. Thomson, C. Zhang, M. Mebrahtu, J. Guss, R. Totoritis, T. Consler, N. Campobasso, D. Taylor, T. Lewis, K. Weaver, M. Mülbaier, J. Seal, R. Dunham, W. Kazmierski, D. Favre, G. Bergamini, L. Shewchuk, A. Rendina and G. Zhang, ChemBioChem, 2020, DOI: 10.1002/cbic.202000298.

11 D. Svatunek, C. Denk, V. Rosecker, B. Sohr, C. Hametner, G. Allmaier, J. Fröhlich and H. Mikula, Monatshefte Für Chem. - Chem. Mon., 2016, 147, 579-585.

12 M. Royzen, G. P. A. Yap and J. M. Fox, J. Am. Chem. Soc., 2008, 130, 3760-3761.

13 Y. Inoue, S. Takamuku, Y. Kunitomi and H. Sakurai, J. Chem. Soc. Perkin Trans. 2, 1980, 1672-1677.

14 L. Hornyák, N. Dobos, G. Koncz, Z. Karányi, D. Páll, Z. Szabó, G. Halmos and L. Székvölgyi, Front. Immunol., 2018, 9, 151. 